\title{
Efeitos da musicoterapia sobre os sintomas de ansiedade e depressão em adultos com diagnóstico de transtornos mentais: revisão sistemática
}

Effects of music therapy on anxiety and depression symptoms in adults

diagnosed with mental disorders: a systematic review

Efectos de la musicoterapia sobre los síntomas de ansiedad y depresión en adultos con diagnóstico de trastornos mentales: revisión sistemática

Aline Raquel de Sousa Ibiapina ${ }^{1}$ iD https://orcid.org/0000-0003-1373-3564 Luís Carlos Lopes-Junior ${ }^{2}$ io https://orcid.org/0000-0002-2424-6510 Lorena Uchôa Portela Veloso ${ }^{3}$ iD https://orcid.org/0000-0002-8062-3624

Ana Paula Cardoso Costa ${ }^{3}$ io nttps://orcid.org/0000-0002-1550-3685 Fernando José Guedes da Silva Júnior ${ }^{3}$ io https://orcid.org//0000-0001-5731-632X Jaqueline Carvalho e Silva Sales ${ }^{3}$ io hitps://orcid.org/0000-0001-7657-5829 Claudete Ferreira de Souza Monteiro ${ }^{3}$ io https://orcid.org/0000-0003-0902-3340

Como citar:

Ibiapina AR, Lopes-Junior LC, Veloso LU, Costa AP, Silva Júnior FJ, Sales JC, et al. Efeitos da musicoterapia sobre os sintomas de ansiedade e depressão em adultos com diagnóstico de transtornos mentais: revisão sistemática. Acta Paul Enferm. 2022;35:eAPE002212.

DOI

http://dx.doi.org/10.37689/acta-ape/2022AR02212

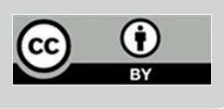

Descritores Musicoterapia; Ansiedade; Depressão; Adulto; Saúde mental

Keywords Music therapy; Anxiety; Depression; Adult; Mental health

Descriptores

Musicoterapia; Ansiedad; Depresión; Adulto; Salud mental

Submetido 11 de Agosto de 2020

Aceito

31 de Maio de 202

Autor correspondente Fernando José Guedes da Silva Júnio E-mail: fernandoguedes@ufpi.edu.br

Editor Associado (Avaliação pelos pares): Alexandre Pazetto Balsanell (https://orcid.org/0000-0003-3757-1061) Escola Paulista de Enfermagem, Universidade Federal de São Paulo, São Paulo, SP, Brasi

\section{Resumo}

Objetivo: Identificar e sintetizar as evidências oriundas de ensaios clínicos randomizados que avaliaram os efeitos da musicoterapia sobre os sintomas de ansiedade e de depressão em adultos com transtornos mentais.

Métodos: Revisão sistemática guiada pelo protocolo Preferred Reporting Items for Systematic Reviews and Meta-Analysis. A busca dos artigos nas bases de dados MEDLINE, Embase, CENTRAL Cohcrane, CINAHL, PsycINF0 e LILACS, bem como a busca manual, seleção dos estudos, extração dos dados e avaliação metodológica pela Escala de Jadad e pela ferramenta do Risco de Viés da Cochrane foram realizadas por dois revisores de forma independente.

Resultados: Foram identificados 1649 estudos, sendo oito selecionados e analisados. Os achados mostraram um efeito positivo da musicoterapia nos sintomas de ansiedade e depressão na população adulta em diferentes contextos. De acordo com a Risco de Viés da Cochrane, quatro estudos foram classificados como baixo risco de viés, e os outros quatro como alto risco de viés. Os adultos que receberam musicoterapia apresentaram relaxamento físico e mental, redução dos sintomas ansiosos e depressivos, promovendo o bem-estar de forma consciente e saudável.

Conclusão: A musicoterapia se apresenta como uma potencial estratégia para reduzir a ansiedade e a depressão bem como para auxiliar na manutenção e recuperação da saúde das pessoas adultas com transtornos mentais.

\section{Abstract}

Objective: To identify and synthesize evidence from randomized clinical trials that assessed the effects of music therapy on anxiety and depression symptoms in adults with mental disorders.

Methods: This is a systematic review guided by the Preferred Reporting Items for Systematic Reviews and Meta-Analysis protocol. The search for articles in the MEDLINE, Embase, CENTRAL Cohcrane, CINAHL, PsycINFO and LILACS databases, as well as manual search, selection of studies, data extraction and methodological assessment using the Jadad Scale and the Cochrane risk-of-bias, was performed by two reviewers independently.

Results: 1,649 studies were identified, eight of which were selected and analyzed. The findings showed a positive effect of music therapy on anxiety and depression symptoms in the adult population in different contexts. According to the Cochrane risk-of-bias, four studies were classified as low risk of bias, and the other four as high risk of bias. Adults who received music therapy showed physical and mental relaxation, reduced anxiety and depressive symptoms, promoting well-being in a conscious and healthy way. 
Conclusion: Music therapy presents itself as a potential strategy to reduce anxiety and depression as well as to help maintain and recover the health of adults with mental disorders.

\section{Resumen}

Objetivo: Identificar y sintetizar las evidencias oriundas de ensayos clínicos aleatorizados que evaluaron los efectos de la musicoterapia sobre los síntomas de ansiedad y depresión en adultos con trastornos mentales.

Métodos: Revisión sistemática orientada por el protocolo Preferred Reporting Items for Systematic Reviews and Meta-Analysis. La búsqueda de los artículos en las bases de datos MEDLINE, Embase, CENTRAL Cohcrane, CINAHL, PsycINF0 y LILACS fue realizada por dos revisores de forma independiente, así como también la búsqueda manual, selección de los estudios, extracción de datos y evaluación metodológica a través de la Escala de Jadad y la herramienta de riesgo de sesgo de Cochrane.

Resultados: Se identificaron 1649 estudios, de los cuales ocho fueron seleccionados y analizados. Los resultados mostraron un efecto positivo de la musicoterapia en los síntomas de ansiedad y depresión en la población adulta en diferentes contextos. De acuerdo con el riesgo de sesgo de Cochrane, cuatro estudios fueron clasificados con bajo riesgo de sesgo y otros cuatro con alto riesgo de sesgo. Los adultos que recibieron musicoterapia presentaron relajamiento físico y mental, reducción de los síntomas ansiosos y depresivos, lo que promueve el bienestar de forma consciente y saludable.

Conclusión: La musicoterapia se presenta como una estrategia potencial para reducir la ansiedad y la depresión, así como para ayudar a mantener y recuperar la salud de las personas adultas con trastornos mentales.

\section{Introdução}

A Organização Mundial da Saúde (OMS) demonstra preocupação significativa com crescimento da população afetada por transtornos mentais. Esse fenômeno atinge cerca de 700 milhóes de pessoas em todo mundo e inclui transtornos como ansiedade e depressão. Dados epidemiológicos sinalizam que a depressão afeta $4,4 \%$ da população global, sendo maior entre as mulheres do que nos homens (5,1\% e 3,6\%, respectivamente); e ansiedade, com prevalência de 3,6\% da populaçáo mundial. ${ }^{(1)}$

A depressão é marcada pela presença de tristeza, anedonia, sentimentos de culpa, falta de autoestima, alterações no sono e no apetite, falta de concentração e cansaço. Já a ansiedade é caracterizada por sentimentos desagradáveis, preocupação e tensão associados a sintomas físicos como agitação, palpitaçôes, calafrios, sudorese, nervosismo. Esses transtornos mentais são crônicos, recorrentes e podem ser classificadas em leves, moderadas ou graves. ${ }^{(1,2)}$ Ambos os transtornos têm efeito devastador na vida dos indivíduos em tratamento de longo prazo, pois, reduzem o incentivo à continuidade do tratamento, aumentam a frequência de hospitalizaçóes, desencadeiam mudanças no estilo de vida, e prejudicam a adesão ao processo terapêutico. ${ }^{(3,4)}$

Nesse contexto, pacientes que sofrem de transtornos mentais podem se beneficiarem de terapias complementares, a exemplo da musicoterapia, para tratamento de sintomas desagradáveis. ${ }^{(5-8)}$ Adicionalmente, a musicoterapia também é recomendada pela
Classificação de Intervençôes de Enfermagem (NIC) como intervenção que auxilia em mudanças no comportamento, resultando em alívio desses sintomas. ${ }^{(9)} \mathrm{A}$ musicoterapia funciona em áreas em que medicamentos não têm eficácia desejada. ${ }^{(10)}$ Pesquisas tem mostrado que a musicoterapia modula respostas imunológicas em adultos, evidenciado por aumento significativo no número de linfócitos, células T, CD4s +, células NK, bem como diminuição de citocinas pró-inflamatórias, tais como: IFN- $\gamma$ e IL-6. ${ }^{(11-14)}$ Ademais a musicoterapia tem sido apontada como boa estratégia para tratamento dos sintomas de doenças, bem como para relação enfermeiro/paciente, tornando o atendimento mais próximo. ${ }^{(10)}$ Trata-se de uma abordagem terapêutica de baixo custo e de fácil acesso que facilita o atendimento biopsicossocial e espiritual, restaura o equilíbrio e o bem-estar, favorece a comunicação, a integração do indivíduo na comunidade. (5,6,15-17) $^{-}$

No Brasil, ao longo do tempo, o Ministério da Saúde ampliou o espectro de práticas integrativas e complementares incluindo a musicoterapia no rol das pertencentes à Política Nacional de Práticas Integrativas e Complementares (PNPIC) no âmbito do Sistema e Único de Saúde. Por meio da Portaria no 849 de 27 de março de 2017, foram incluídas: a arteterapia, ayurveda, biodança, dança circular, meditação, musicoterapia, naturopatia, osteopatia, quiropraxia, reflexoterapia, reiki, shantala, terapia comunitária integrativa e o yoga. ${ }^{(18)}$ Com a portaria no 702 de 21 de março de 2018, alterou-se a Portaria de Consolidação no 2/GM/MS, de 28 de setembro de 2017, para incluir outras novas práticas 
na PNPIC, quais sejam, aromaterapia, apiterapia, bioenergética, constelação familiar, cromoterapia, geoterapia, hipnoterapia, imposição de mãos, medicina antroposófica/antroposofia aplicada à saúde, ozonioterapia, terapia de florais e termalismo social/ crenoterapia. Dessa forma, o SUS autoriza, atualmente, 29 práticas integrativas e complementares, intensificando o desafio da capacitação, implantação e oferta destas na saúde pública do país.

Uma recente revisão sistemática com meta-análise da Cochrane sobre a temática que objetivou avaliar os efeitos da musicoterapia para a depressão na população em geral em comparação com o tratamento usual encontrou evidências de amplos efeitos de qualidade moderada à favor da musicoterapia quando comparado com o tratamento usual sozinho, para ambos os sintomas depressivos avaliados pelo médico (SMD -0,98, IC 95\% -1,69 a -0,27, $\mathrm{n}=219)$ e sintomas depressivos relatados pelo paciente (SMD -0,85, IC de 95\% - 1,37 a -0,34, $\mathrm{n}=$ 142). ${ }^{(19)}$ Contudo, a eficácia da musicoterapia particularmente, nos sintomas de ansiedade e depressão em adultos que receberam diagnósticos de transtornos mentais ainda não foi elucidada. Portanto, estudo que sintetize evidências dos efeitos da musicoterapia, especificamente, em adultos - principal grupo acometido pelas consequências desses sintomas de ansiedade e depressão, poderá auxiliar profissionais de saúde na tomada de decisão e implementação dessa terapia complementar na prática clínica. Nesse sentido, tem-se como objetivo identificar e sintetizar evidências oriundas de ensaios clínicos randomizados que avaliaram os efeitos da musicoterapia sobre os sintomas de ansiedade e de depressão em adultos com transtornos mentais.

\section{Métodos}

Estudo de revisão sistemática de ensaios clínicos randomizados (ECR) guiado pelo Preferred Reporting Items for Systematic Reviews and MetaAnalyses (PRISMA). ${ }^{(20)}$ Para formular o objetivo e a pergunta da revisão foi utilizada a estratégia PICOS (P - Population or Pacientes; I - Intervention; $C$ - Comparison; O - Outcomes; S - Study design), onde População (P): adultos acometidos por transtornos mentais; Intervenção (I): musicoterapia; Comparação / controle $(\mathrm{C})$ : tratamento padrão e Desfecho $(\mathrm{O})$ : redução dos sintomas de ansiedade e depressão; Desenho do estudo (S): apenas ensaios clínicos randomizados. ${ }^{(21)}$ Obteve-se, assim, a seguinte pergunta dessa revisão: Qual o efeito da musicoterapia sobre sintomas de ansiedade e de depressão em adultos que sofrem de transtornos mentais?

Os critérios de inclusão estabelecidos foram: estudos primários cujo delineamento fosse o ensaio clínico randomizado (ECR), realizados com adultos ( $\geq 18$ anos), de ambos os sexos, com diagnóstico/ avaliação de sintomas de ansiedade ou depressão, que reportassem uso da musicoterapia, ativa ou passiva, como intervençáo. Os critérios de exclusão, por sua vez, foram: toda literatura cinzenta, dissertaçóes, teses, editoriais, estudos de protocolo, diretrizes clínicas, estudos cujos participantes foram crianças ou idosos e estudos que utilizaram mais de uma terapia complementar em combinação.

A busca pelos estudos foi realizada em nove de dezembro de 2019, nas seguintes bases de dados: Medical Literature Analysis and Retrieval System Online (MEDLINE) via PubMed, Embase (Excerpta Medica database), CENTRAL Cohcrane, Cumulative Index to Nursing and Allied Health Literature (CINAHL), Literatura Latino-Americana do Caribe em Ciências da Saúde (LILACS) e American Psychological Association (APA) PsycINFO. Não foram aplicados limites de temporalidade de publicação e nem de idioma.

Inicialmente, a estratégia para busca dos estudos foi composta por uma combinação de descritores controlados (indexadores nas respectivas bases de dados) e palavras-chave, segundo indicação oferecida em cada base de dado eletrônica. Assim, para busca dos artigos na MEDLINE, foram usados descritores controlados do Medical Subject Headings (MeSH); o PsycINFO Thesaurus foi consultado para a base PsycINFO; o Emtree terms para a Embase; e os CINAHL subject headings para a base CINAHL. De modo a ampliar estratégia de busca, foi realizada uma combinaçáo dos descritores controlados e palavras-chave por meio de operadores booleanos "AND" e "OR"(22-25) para obter combinações restri- 
tiva e aditiva, respectivamente. Além disso, a busca foi realizada utilizando-se descritores identificados e com sentido ampliado, sem o uso dos filtros das bases de dados para preservar amostras significativas e garantir menor risco de perdas. Essa estratégia justifica o número reduzido de estudos selecionados diante da amostra obtida, somada ao fato de estabelecermos como critério o ECR como delineamento para abarcarmos as evidências mais fortes para tomada de decisão na prática clínica. $\mathrm{O}$ quadro 1 apresenta a estratégia de busca final processada nas respectivas bases de dados.

Quadro 1. Expressão de busca nas bases de dados pesquisadas

\begin{tabular}{|c|c|}
\hline Base de dados & Expressão da busca \\
\hline $\begin{array}{l}\text { MEDLINE*/ } \\
\text { PubMed }\end{array}$ & $\begin{array}{l}\text { ("adult" [MeSH† Terms]) AND ("depressive disorder" [MeSH† Terms] } \\
\text { OR ("depressive"[All Fields] AND "disorder"[All Fields]) OR "depressive } \\
\text { disorder"[All Fields] OR "depression"[All Fields] OR "depression"[MeSH† } \\
\text { Terms]) AND ("anxiety"[MeSH† Terms] OR "anxiety"[All Fields]) AND } \\
\text { ("music therapy"[MeSH † Terms] OR ("music"[All Fields] AND "therapy"[All } \\
\text { Fields]) OR “music therapy"[All Fields]) }\end{array}$ \\
\hline 挨mbase & adult AND depression OR anxiety OR 'music therapy' \\
\hline $\begin{array}{l}\text { §CENTRAL } \\
\text { Cohcrane }\end{array}$ & $\begin{array}{l}\text { ("adult" [MeSH† Terms]) AND ("depressive disorder"[MeSH Terms] OR } \\
\text { ("depressive"[All Fields] AND "disorder"[All Fields]) OR "depressive } \\
\text { disorder"[All Fields] OR "depression"[All Fields] OR "depression"[MeSH } \\
\text { Terms]) AND (“anxiety"[MeSH Terms] OR "anxiety"[All Fields]) AND (“music } \\
\text { therapy"[MeSH Terms] OR (“music"[All Fields] AND "therapy"[All Fields]) } \\
\text { OR “music therapy"[All Fields]) }\end{array}$ \\
\hline "PsycINF0 & $\begin{array}{l}\text { adult [Thesaurus] AND depression [Thesaurus] OR anxiety [Thesaurus] } \\
\text { AND music therapy [Thesaurus] }\end{array}$ \\
\hline${ }^{\star \star} \mathrm{CINAHL}$ & adult AND depression OR anxiety AND music therapy \\
\hline 扎ILACS & $\begin{array}{l}\text { adulto OR adult OR adulto AND depressão OR depression OR depresión } \\
\text { [Palavras] AND ansiedade OR anxiety OR ansiedad [Palavras] AND } \\
\text { musicoterapia OR music therapy OR musicoterapia [Palavras] }\end{array}$ \\
\hline
\end{tabular}

Além das bases de dados eletrônicas citadas acima, foram realizadas buscas secundárias em outras fontes diversas, tais como em sites de Registros de Ensaios Clínicos, como, por exemplo, o ClinicalTrials.gov (National Institutes of Health, NIH, USA), The Brazilian Clinical Trials Registry (via Plataforma ReBEC) e no Google Scholar. Além disso, a lista de referências finais que constava nos estudos primários incluídos foi analisada manualmente a fim de encontrar estudos relevantes a serem adicionados. ${ }^{(23,24)}$ Vale ressaltar que dois pesquisadores realizaram a estratégia de busca de forma independente. $\mathrm{O}$ software bibliográfico EndNote ${ }^{\oplus}$ (https://www.myendnoteweb.com/) foi usado para armazenar, organizar e gerenciar todas as referências e garantir uma pesquisa sistemática e abrangente.
A amostra foi selecionada por dois revisores, de maneira independente e às cegas. Após essa seleção, um terceiro revisor foi responsável por analisar e decidir (junto com os anteriores) sobre a inclusão ou exclusão de cada artigo, principalmente em relação àqueles contendo decisão conflitante. Posteriormente, à seleçâo do terceiro revisor, uma busca manual foi feita a partir das referências dos artigos selecionados.

Os dados foram extraídos baseados em ferramentas pré-estabelecidas ${ }^{(25-30)}$ e incluíram quatro domínios: I) identificação do estudo, com dados como título do artigo, fator de impacto do periódico, país dos autores do estudo, ano de publicação, instituição anfitriã do estudo (hospital; universidade; centro de pesquisa; estudo multicêntrico ou estudo em uma única instituição); conflitos de interesse; financiamento; II) características metodológicas (delineamento do estudo; objetivo do estudo ou questão de pesquisa ou hipóteses; características da amostra), por exemplo, tamanho da amostra, idade, características do baseline dos grupos experimentais e controle, método de recrutamento, perdas, duração do acompanhamento, análises estatísticas; III) principais achados e implicação para a prática clínica; e IV) conclusóes.

Para a extração dos dados, foram elaborados dois quadros por meio do Microsoft Word ${ }^{\circledR}$, por dois pesquisadores de modo independente para sintetizar os dados dos estudos incluídos. Após essa fase, os quadros foram compilados em um único para prosseguir com as análises qualitativa.

A qualidade metodológica dos ECR foi avaliada pela Escala de Jadad, cuja pontuação varia de 0 a 5 , com estudos de pontuação $<3$ considerados de baixa qualidade e estudos com pontuação $\geq 3$ classificados como de alta qualidade. ${ }^{(31)}$ A validade interna e o risco de viés para os ECR foram avaliados utilizando a ferramenta do Risco de Viés da Cochrane do Handbook da Colaboração Cochrane para Revisões Sistemáticas de Intervenções, versão 5.1.0 (RoB 1), ${ }^{(32)}$ a qual avalia os sete domínios: I) Alocação da sequência de randomização (viés de seleção); II) Sigilo da alocação (viés de seleção); III) Cegamento dos participantes e equipe envolvida (viés de performance); IV) Cegamento de avalia- 
dores de desfecho (viés de detecção); V) Desfechos incompletos (viés de atrito); VI) Relato de desfecho seletivo (viés de publicação) e VII) Outras fontes de viés. Com base nesses domínios avaliados, os estudos são classificados em risco de viés baixo, alto ou incerto.

Uma vez que, a maioria dos estudos avaliados apresentaram diferenças metodológicas significativas, optou-se por realizar uma síntese qualitativa dos dados na presente revisão sistemática.

\section{Resultados}

O levantamento nas bases de dados resultou em 1.649 estudos, e nenhum estudo adicional foi incluído após pesquisas no Google Scholar, bem como nos registros de ensaios clínicos e nas referências dos artigos primários selecionados. A triagem da busca revelou 991 duplicatas, resultando em 658 registros após a remoção das duplicações. A primeira triagem, baseada nos critérios de exclusão, bem como através da leitura de títulos e resumos, excluiu a maioria dos estudos (625). Após a elegibilidade/ avaliação crítica dos textos completos de 33 registros, 8 registros atenderam a todos os critérios de inclusão e prosseguiram para a fase de extração de dados, leitura exaustiva e síntese qualitativa. A figura 1 apresenta um fluxograma do processo de busca de acordo com o fluxograma PRISMA. ${ }^{(20)}$

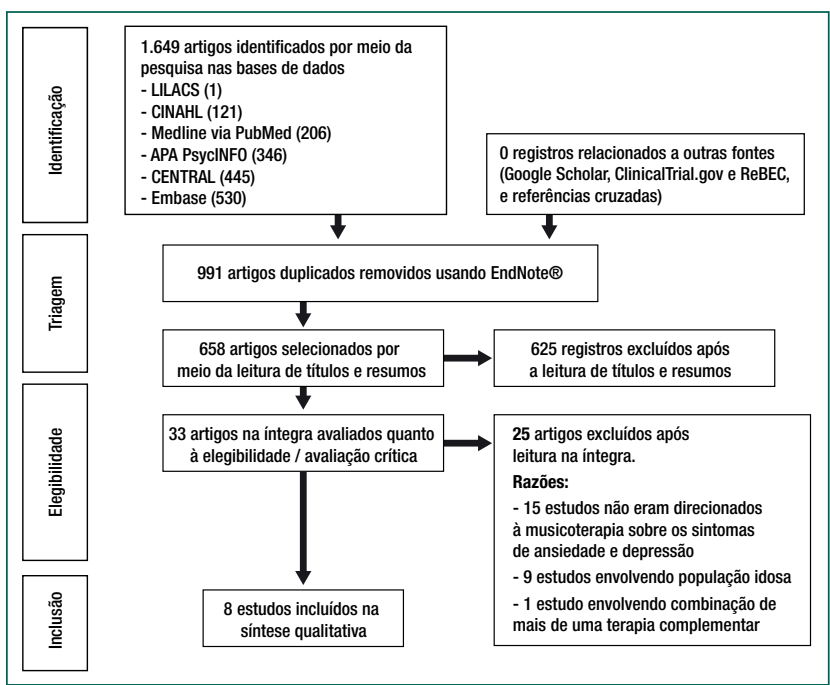

Figura 1. Fluxograma PRISMA ${ }^{(20)}$ para seleção doa estudos
O quadro 2 resume as principais características dos estudos incluídos ${ }^{(33-40)}$ na síntese qualitativa em ordem cronológica. Os estudos foram realizados em oito países diferentes, incluindo Brasil, ${ }^{(39)}$ Canadá, ${ }^{(40)}$ Finlândia, ${ }^{(35)}$ França, ${ }^{(36)}$ Irã, ${ }^{(38)}$ Noruega, ${ }^{(37)}$ Coréia do Sul, ${ }^{(34)}$ e EUA. ${ }^{(33)}$ Todos os estudos foram realizados em centros únicos e a maioria incluía ambos os sexos. ${ }^{(33-36,38-40)}$ Um único estudo incluiu apenas homens, ${ }^{(37)}$ uma vez que a população do estudo era composta por adultos privados de liberdade. A maioria dos estudos $(n=7)$ incluiu adultos com transtornos mentais com diferentes comorbidades. ${ }^{(33-36,38-40)}$ A média de participantes que compuseram as amostras nos estudos da foi de 75 (valor mínimo de 26 e valor máximo de 113 participantes).

Ansiedade e depressão foram os desfechos primários analisados na maioria dos estudos incluídos $(\mathrm{n}=7) .^{(33-35,37-40)}$ Outros desfechos avaliados foram dor; ${ }^{(36,39)}$ humor, ${ }^{(33,39)}$ qualidade de vida, ${ }^{(35)}$ relaçôes sociais $^{(34,37)}$ e deficiência/incapacidade. ${ }^{(40)}$

Três estudos avaliaram a ansiedade através do State Trait Anxiety Inventory - STAI ${ }^{(34,37,38)}$ e outros três estudos avaliaram a ansiedade pela Escala Hospitalar de Ansiedade e Depressão-HADS . ${ }^{(35,36,40)}$ A depressão foi avaliada utilizando a Escala Hospitalar de Ansiedade e Depressão - HADS em cinco estudos; ; $^{(34-37,40)}$ e apenas um estudo utilizou o Beck Depression Inventory BDI para avaliar os sintomas depressivos. ${ }^{(38)}$ A dor foi avaliada pela Escala Visual Analógica - EVA. ${ }^{(36,39)}$ Além disso, o humor foi avaliado pelo Profile of Mood States - POMS; ${ }^{(33,39)}$ qualidade de vida relacionado à saúde pelo Health-related Quality of Life Survey RAND-36, ${ }^{(35)}$ relaçóes sociais pelo Questionário de Qualidade de Vida e Apreciação e Satisfação - Q-LES-Q ${ }^{(37)}$ ou usando a escala de mudança de relacionamento - RCS. ${ }^{(34)}$ Um estudo avaliou a incapacidade como resultado usando o Programa de Avaliação de Deficiência da OMS 2.0. ${ }^{(40)}$

Entre os oito ECR, $(n=4 ; 50 \%)$ apresentou alta qualidade metodológica ${ }^{(35,36,39,40)}$ de acordo com a Escala de Jadad (escores 3 ou 4) (Quadro 2).

Em relação ao risco de viés de acordo com a RoB 1 para ECR, quatro estudos ${ }^{(35,36,39,40)}$ foram classificados como baixo risco de viés, e os outros quatro $^{(33,34,37,38)}$ como alto risco de viés (Figura 2). ${ }^{(32)}$ 
Quadro 2. Caracterização dos oito artigos selecionados segundo autores, país, objetivo, amostra, intervenção, variáveis, instrumentos, principais resultados e escore conforme Escala de Jadad

\begin{tabular}{|c|c|c|c|c|c|c|c|}
\hline \multirow{2}{*}{$\begin{array}{l}\text { Autores / } \\
\text { País }\end{array}$} & \multirow{2}{*}{ Objetivo } & \multirow{2}{*}{ Amostra } & \multicolumn{2}{|c|}{ Intervenção } & \multirow{2}{*}{$\begin{array}{l}\text { Variáveis/ } \\
\text { Instrumentos }\end{array}$} & \multirow{2}{*}{ Principais resultados } & \multirow{2}{*}{$\begin{array}{l}\text { Escore } \\
\text { Jadad }\end{array}$} \\
\hline & & & Grupo Experimental (GE) & $\begin{array}{l}\text { Grupo Controle } \\
\text { (GC) }\end{array}$ & & & \\
\hline $\begin{array}{l}\text { Cassileth et } \\
\text { al. } 2003^{(33)} \\
\text { EUA }\end{array}$ & $\begin{array}{l}\text { Determinar os efeitos da } \\
\text { musicoterapia sobre o } \\
\text { humor em comparação com } \\
\text { o tratamento padrão durante } \\
\text { internações para terapia de } \\
\text { altas doses com transplante } \\
\text { autólogo de células-tronco }\end{array}$ & $\begin{array}{l}69 \text { pacientes } \\
\text { com malignidade } \\
\text { hematológica } \\
\text { admitidos para } \\
\text { terapia de } \\
\text { alta dose com } \\
\text { transplante } \\
\text { autólogo de } \\
\text { células-tronco } \\
\end{array}$ & \begin{tabular}{|l|} 
(n=36) alocados para 0 \\
grupo de musicoterapia \\
- Intervenção: 20 a 30 \\
minutos; sessões; $2-3$ vezes \\
/ semana); Seguimento: 6 \\
meses
\end{tabular} & $\begin{array}{l}(\mathrm{n}=33) \text { alocados } \\
\text { para tratamento } \\
\text { padrão sem } \\
\text { musicoterapia }\end{array}$ & $\begin{array}{l}\text { Humor } \\
\text { - POMS* }\end{array}$ & $\begin{array}{l}\text { Durante a internação, os pacientes } \\
\text { do GE (musicoterapia) tiveram uma } \\
\text { pontuação } 28 \% \text { menor na escala de } \\
\text { Ansiedade / Depressão ( } p=0,065 \text { ) e } \\
37 \% \text { menor ( } p=0,01 \text { ) na pontuação total } \\
\text { de distúrbios de humor em comparação } \\
\text { aos pacientes do GC }\end{array}$ & 2 \\
\hline $\begin{array}{l}\text { Choi et al. } \\
2008^{(34)} \\
\text { Coréia do } \\
\text { Sul }\end{array}$ & $\begin{array}{l}\text { Testar a eficácia da } \\
\text { musicoterapia em grupo } \\
\text { para melhorar a depressão, } \\
\text { ansiedade e relacionamentos } \\
\text { em pacientes psiquiátricos }\end{array}$ & $\begin{array}{l}26 \text { pacientes } \\
\text { psiquiátricos }\end{array}$ & $\begin{array}{l}\text { (n=13) alocados para } 0 \\
\text { grupo de musicoterapia } \\
\text { - Intervenção: } 60 \text { minutos; } \\
15 \text { sessões (uma vez até } \\
\text { duas vezes por semana); } \\
\text { Seguimento: } 15 \text { semanas }\end{array}$ & $\begin{array}{l}(n=13) \text { alocados } \\
\text { para o tratamento } \\
\text { padrão sem } \\
\text { musicoterapia }\end{array}$ & $\begin{array}{l}\text { Ansiedade } \\
\text { - STAl } \\
\text { Depressão } \\
\text { - HADS }{ }^{\ddagger} \\
\text { Relações Sociais } \\
\text { - RCS }\end{array}$ & $\begin{array}{l}\text { Após } 15 \text { sessões, a intervenção de } \\
\text { musicoterapia em grupo melhorou } \\
\text { significativamente as pontuações para } \\
\text { depressão }(F[1,25]=257,7 ; p=0,001) \text {, } \\
\text { ansiedade }(F[1,25]=202,7 ; p=0,001) \text {, } \\
\text { e relacionamentos sociais }(F[1,25] \\
=450,6 ; p=0,001) \text { em pacientes } \\
\text { psiquiátricos em comparação com o GC }\end{array}$ & 1 \\
\hline $\begin{array}{l}\text { Erkkilä et al. } \\
2011^{(35)} \\
\text { Finlândia }\end{array}$ & $\begin{array}{l}\text { Determinar a eficácia da } \\
\text { musicoterapia adicionada ao } \\
\text { atendimento usual padrão } \\
\text { comparado com apenas o } \\
\text { atendimento usual padrão no } \\
\text { tratamento da depressão } \\
\text { entre pessoas em idade ativa }\end{array}$ & $\begin{array}{l}79 \text { adultos com } \\
\text { depressão unipolar }\end{array}$ & $\begin{array}{l}\text { ( } n=33) \text { pacientes alocados } \\
\text { a receberem musicoterapia e } \\
\text { tratamento padrão } \\
\\
\text { - Intervenção: } 60 \text { minutos; } \\
20 \text { sessões (duas vezes por } \\
\text { semana); } \\
\text { Seguimento: } 6 \text { meses }\end{array}$ & $\begin{array}{l}\text { ( } \mathrm{n}=46) \text { pacientes } \\
\text { alocados apenas } \\
\text { para tratamento } \\
\text { padrão sem } \\
\text { musicoterapia }\end{array}$ & $\begin{array}{l}\text { Ansiedade e } \\
\text { Depressão } \\
\text { - MADRS } \\
\text { - HADS } \ddagger \\
\text { - GAF* } \\
\text { Qualidade de Vida } \\
\text { - RAND36 }{ }^{\star \dagger}\end{array}$ & 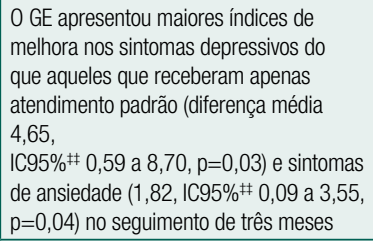 & 3 \\
\hline $\begin{array}{l}\text { Guétin et al. } \\
2012^{(36)} \\
\text { França }\end{array}$ & $\begin{array}{l}\text { Avaliar a utilidade da } \\
\text { musicoterapia no tratamento } \\
\text { de pacientes com dor } \\
\text { crônica }\end{array}$ & \begin{tabular}{|l}
87 \\
pacientes com \\
dor lombar, \\
fibromialgia, \\
doença \\
inflamatória \\
ou doença \\
neurológica \\
\end{tabular} & $\begin{array}{l}\text { ( } \mathrm{n}=44 \text { ) alocados ao grupo de } \\
\text { musicoterapia } \\
\text { - Intervenção: } 20 \text { minutos; } \\
\text { (duas vezes ao dia); } \\
\text { Seguimento: } 3 \text { meses }\end{array}$ & $\begin{array}{l}(\mathrm{n}=43 \text { ) alocados no } \\
\text { grupo de tratamento } \\
\text { padrão sem } \\
\text { musicoterapia }\end{array}$ & $\begin{array}{l}\text { Dor } \\
\text { - VAS" } \\
\text { Ansiedade e } \\
\text { Depressão } \\
\text { - HADS }{ }^{\ddagger}\end{array}$ & $\begin{array}{l}\text { No GE (musicoterapia), houve redução } \\
\text { mais significativa da dor ( } \mathrm{p}<0,001 \text { ) } \\
\text { quando comparado ao GC. Além } \\
\text { disso, a musicoterapia contribuiu para } \\
\text { reduzir significativamente a ansiedade, } \\
\text { depressão e o consumo de agentes } \\
\text { ansioliticos }\end{array}$ & 3 \\
\hline $\begin{array}{l}\text { Gold et al. } \\
2014^{(37)} \\
\text { Noruega }\end{array}$ & $\begin{array}{l}\text { Examinar os efeitos } \\
\text { da musicoterapia nos } \\
\text { aspectos da saúde mental, } \\
\text { especificamente depressão, } \\
\text { ansiedade e relacionamento } \\
\text { social em adultos privados } \\
\text { de liberdade }\end{array}$ & $\begin{array}{l}113 \text { homens } \\
\text { adultos privados de } \\
\text { liberdade }\end{array}$ & $\begin{array}{l}\text { ( } \mathrm{n}=56 \text { ) alocados para } \\
\text { musicoterapia } \\
\text { - Intervenção: tempo } \\
\text { não reportado; sessões } \\
\text { (2 a } 3 \text { vezes / semana); } \\
\text { Seguimento: } 6 \text { meses }\end{array}$ & $\begin{array}{l}(\mathrm{n}=57) \text { alocados } \\
\text { para tratamento } \\
\text { padrão sem } \\
\text { musicoterapia }\end{array}$ & $\begin{array}{l}\text { Ansiedade } \\
\text { - STAI } \\
\text { Depressão } \\
\text { - HADS }{ }^{\ddagger} \\
\text { Relações Sociais } \\
\text { - Q-LES-Q\$ }\end{array}$ & $\begin{array}{l}\text { A musicoterapia foi bem aceita pelos } \\
\text { adultos privados de liberdade. A análise } \\
\text { post-hoc das alterações dentro do grupo } \\
\text { experimental sugeriu uma redução } \\
\text { da ansiedade após } 2 \text { semanas de } \\
\text { musicoterapia ( } d=0,33, p=0,025 \text { ) }\end{array}$ & 2 \\
\hline $\begin{array}{l}\text { Salehi et al. } \\
2016^{(38)} \\
\text { Irã }\end{array}$ & $\begin{array}{l}\text { Determinar o efeito } \\
\text { de músicas relaxantes } \\
\text { selecionadas em pacientes } \\
\text { internados com ansiedade } \\
\text { e depressão durante a } \\
\text { hemodiálise }\end{array}$ & $\begin{array}{l}102 \text { pacientes em } \\
\text { hemodiálise }\end{array}$ & $\begin{array}{l}\text { (n=83) receberam } \\
\text { musicoterapia } \\
\text { - Intervenção: } 3 \text { horas; } 1 \\
\text { sessão; } \\
\text { Seguimento: } 1 \text { dia }\end{array}$ & $\begin{array}{l}\text { ( } \mathrm{n}=83) \text { não } \\
\text { receberam } \\
\text { musicoterapia } \\
\text { (atendimento padrão) }\end{array}$ & $\begin{array}{l}\text { Ansiedade } \\
\text {-STAl } \\
\text { Depressão } \\
\text { - BDI"II }\end{array}$ & $\begin{array}{l}\text { Não houve diferenças significativas entre } \\
\text { os grupos antes da hemodiálise BDD|"I' } \\
\text { ( } p=0,253) \text {, t-ansiedade }(p=0,546) \text { e } \\
\text { s-ansiedade ( } p=0,253) \text {, T-Ansiedade } \\
(p=0,546) \text { e } S \text {-Ansiedade }(p=0,776) \text {. } \\
\text { Também não houve diferença significativa } \\
\text { no BDI"' }(p=0,253) \text { e T-ansiedade } \\
\text { ( } p=0,253) \text { e T-Ansiedade ( } p=0,546) \text { entre } \\
\text { os dois grupos após a hemodiálise }\end{array}$ & 2 \\
\hline $\begin{array}{l}\text { Dóro et al. } \\
2017^{(39)} \\
\text { Brasil }\end{array}$ & $\begin{array}{l}\text { Avaliar o efeito da } \\
\text { musicoterapia } \\
\text { sobre a dor, o humor e a } \\
\text { ansiedade }\end{array}$ & \begin{tabular}{|l}
100 \\
pacientes \\
submetidos \\
ao transplante \\
alogênico \\
células-tronco \\
hematopoiéticas
\end{tabular} & $\begin{array}{l}\text { ( } \mathrm{n}=50) \text { pacientes } \\
\text { foram selecionados para a } \\
\text { musicoterapia experimental } \\
\\
\text { - Intervenção: } 30 \text { minutos; } \\
\text { sessões baseadas na } \\
\text { necessidade clínica (duas } \\
\text { vezes por semana); } \\
\text { Seguimento: não reportado }\end{array}$ & $\begin{array}{l}\mathrm{n}=50) \\
\text { pacientes que } \\
\text { receberam apenas } \\
\text { o tratamento padrão } \\
\text { sem musicoterapia }\end{array}$ & $\begin{array}{l}\text { Dor, ansiedade e } \\
\text { humor } \\
\text { - VAS" }\end{array}$ & \begin{tabular}{|l|} 
Foram observadas diferenças \\
significativas na comparação dos grupos, \\
melhorando o humor no GE $(p<0,001)$
\end{tabular} & 4 \\
\hline $\begin{array}{l}\text { Trimmer et } \\
\text { al. } 2018^{40)} \\
\text { Canadá }\end{array}$ & $\begin{array}{l}\text { Testar a viabilidade de uma } \\
\text { música de baixa intensidade } \\
\text { baseada na terapia } \\
\text { cognitivo-comportamental } \\
\text { para sintomas de depressão } \\
\text { e ansiedade }\end{array}$ & \begin{tabular}{|l|}
28 pacientes \\
com sintomas \\
de depressão \\
e ansiedade \\
atendidos em \\
serviço de saúde \\
mental comunitária
\end{tabular} & $\begin{array}{l}\text { (n=14) alocados para o } \\
\text { grupo de intervenção com } \\
\text { musicoterapia } \\
\\
\text { - Intervenção: } 90 \text { minutos; } \\
9 \text { sessões; Seguimento: } 9 \\
\text { semanas }\end{array}$ & $\begin{array}{l}\text { (n=14) alocados } \\
\text { para o grupo de } \\
\text { tratamento padrão } \\
\text { sem musicoterapia }\end{array}$ & 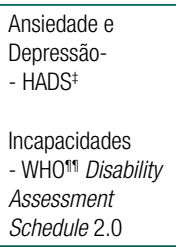 & $\begin{array}{l}\text { Os participantes do GE (musicoterapia) } \\
\text { mostraram melhora na incapacidade } \\
(p=0,027) \text {. Apesar da redução nos } \\
\text { escores de depressão e ansiedade, } \\
\text { essas diferenças não foram } \\
\text { estatisticamente significativas }\end{array}$ & 3 \\
\hline
\end{tabular}

*POMS - Profile of Mood States; 'STAI - State Trait Anxiety Inventory; ${ }^{* H A D S}$ - Hospital Anxiety and Depression Scale; \$RCS - Relationship Change Scale; "VAS - Visual Analog Scale; "MDARS - Montgomery-Asberg Depression Rating Scale; ${ }^{\star \star}$ GAF - Global Assessment of Functioning; ${ }^{+1 R E N D}-36$ - Health-related quality of life survey; ${ }^{\# I C 95 \% ~-~ I n t e r v a l o ~ d e ~ c o n f i a n c ̧ a ~ 95 \% ; ~}{ }^{\S} Q$ Q-LES-Q - Quality of Life Enjoyment and Satisfaction Questionnaire; "'"BDI Beck Depression Inventory; " 


\begin{tabular}{|c|c|c|c|c|c|c|c|c|}
\hline & 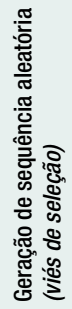 & 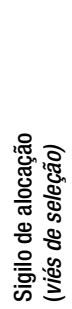 & 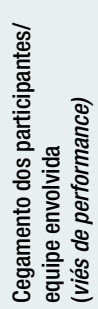 & 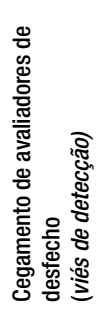 & 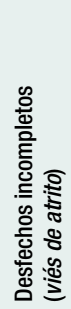 & 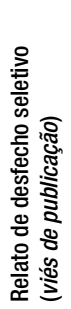 & 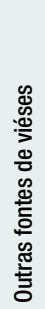 & 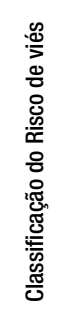 \\
\hline Estudos & \multicolumn{7}{|c|}{ Domínio do Risco de Viés } & \\
\hline Cassileth et al. $2003^{(33)}$ & + & & & & $?$ & + & $?$ & Alto \\
\hline Choi et al. 2008 & 1 & & & & + & $?$ & $?$ & Alto \\
\hline Erkkilä et al. 2011(35) & + & + & & + & + & + & + & Baixo \\
\hline Guétin et al. 2012(36) & + & + & & + & + & + & + & Baixo \\
\hline Gold et al. 2014 & + & + & & $?$ & - & $?$ & $?$ & Alto \\
\hline Salehi et al. 2016 & - & $?$ & & & + & + & $?$ & Alto \\
\hline Dóro et al. 2017(39) & + & + & & + & + & $?$ & + & Baixo \\
\hline Trimmer et al. 2018(40) & + & + & & + & + & + & + & Baixo \\
\hline
\end{tabular}

Fonte: Higgins JPT, Green S. Cochrane Handbook for Systematic Reviews of Interventions. Version 5.1. Cochrane Collaboration; 2011. Avaliable from: https://training.cochrane.org/handbooks ${ }^{(32)}$

*Avaliação da validade interna e do risco de viés dos ensaios clínicos incluídos no estudo segundo a Ferramenta da Colaboração Cochrane (RoB 1) para avaliação do risco de viés de ensaios clínicos randomizados;

†Porcentagem de risco de viés entre os ensaios clínicos por domínios da Ferramenta da Colaboração Cochrane para avaliação do risco de viés de ensaios clínicos randomizados. Símbolo de adição (+) indica baixo risco de

viés; símbolo negativo (-) indica alto risco de viés; ponto de interrogação (?) indica risco incerto de viés

Figura 2. Risco de viés dos oito ensaios clínicos randomizados incluídos e avaliados pela ferramenta da Colaboração Cochrane

O principal viés apresentado por todos os ECR diz respeito ao cegamento de participantes e pessoal (viés de desempenho). Em contrapartida, a maioria dos estudos teve baixo risco de viés envolvendo desfechos incompletos (viés de atrito), relato de desfechos seletivos e outras fontes de vieses.

\section{Discussão}

Os resultados deste estudo contribuem para a ampliação de conhecimentos acerca da temática do uso de terapias complementares no cuidado em saúde. De modo geral, os estudos analisados mostraram um efeito positivo da musicoterapia nos sintomas ansiosos e depressivos na populaçáo adulta com transtornos mentais em vários cenários. Adultos que receberam musicoterapia apresentaram relaxamento físico e mental, redução de sintomas ansiosos e depressivos, promovendo o bem-estar de maneira consciente e saudável. Ademais, os resultados revelaram, ainda, uma taxa significativamente maior de adesão à musicoterapia, principalmente em pacientes adultos hospitalizados.

ECRs bem conduzidos são o padrão-ouro para avaliar intervençôes, pois, seu desenho permite controlar diversos erros sistemáticos tais como, viés de seleção, viés de mensuração e viés de confusão inerentes ao delineamento ${ }^{(41,42)}$ Com relação à validade interna dos estudos incluídos em nossa revisão, quatro estudos (50\%) foram classificados como baixo risco de viés. Esses achados são diferentes dos resultados obtidos em uma recente revisão sistemática e meta-análise que objetivou determinar a eficácia da musicoterapia no tratamento da depressão em idosos. ${ }^{(5)}$ Com base na avaliação da validade interna dos estudos usando a ferramenta de risco de viés da Cochrane (RoB 1), verificou-se que a maioria dos estudos foi classificada como alto risco de viés devido principalmente a métodos de randomização pouco claros; métodos de cegamento não apropriados e presença de viés de atrito. ${ }^{(5)}$ Comumente o viés de performance se apresenta como limitante em ensaios clínicos que utilizam de terapias complementares, seja pelo fato da dificuldade de cegamento dos participantes devido por exemplo a natureza da intervenção como parte integrante da rotina do serviço de saúde, e ou dificuldades no cegamento dos avaliadores de desfechos.

Depressão e ansiedade são doenças incapacitantes que causam sérios problemas, com consequente redução da qualidade de vida dos indivíduos. Nesse sentido, articular a musicoterapia com o tratamento convencional ou atendimento padrão, é visto 
como uma oportunidade de entrar em contato com as emoçóes e promover a interpessoalidade entre o profissional de saúde e os pacientes. ${ }^{(10)}$ Essa terapia complementar demonstra que, além de proporcionar distração, torna-se um meio de comunicação capaz de superar barreiras e limites da expressão verbal. ${ }^{(8,15,40,43,44)}$

A depressão é outra questão emocional identificada como resultado da não adaptação a ambientes e programas correcionais. Um dos estudos analisados nesta revisão ${ }^{(37)}$ apontou que os problemas de saúde mental são predominantes entre adultos privados de liberdade, especificamente ansiedade e depressão. Esses achados estão em linha com um estudo no qual adultos privados de liberdade, com antecedentes criminais menores, de uma unidade penitenciária na China, que haviam participado de musicoterapia com atividades de educação em saúde, atendimento e aconselhamento individual, mostraram redução dos sintomas de ansiedade, depressão e aumento da autoestima. Essa prática favoreceu o controle dos impulsos e da raiva. Além disso, para os adultos privados de liberdade, que não foram capazes de expressar ou discutir seus problemas através de um diálogo verbal, a música serviu como um meio para ajudar a liberar emoçóes e pensamentos de uma maneira positiva. ${ }^{(45)}$

Nesta revisão, foram reunidos estudos que mostraram efeitos da musicoterapia sobre a ansiedade e a depressão, em indivíduos adultos com diferentes doenças crônicas com transtornos mentais associados, como insuficiência renal crônica, ${ }^{(38)}$ câncer, ${ }^{(33,39)}$ depressão ${ }^{(34,35)}$ e dor crônica. ${ }^{(36)}$ Indivíduos e cuidadores no tratamento do câncer lidam com o isolamento, distúrbios emocionais, ansiedade, transtornos do humor, mudanças na cognição e sintomas de depressão. ${ }^{(4)}$ Em relação ao impacto da musicoterapia em pacientes com crônicos incluindo o câncer, resultados científicos que mostram os benefícios psicológicos e fisiológicos na população adulta e pediátrica. ${ }^{(7,15,23,46-51)}$

A musicoterapia também tem um impacto fisiológico e emocional positivo em pessoas que estão sob tratamento de trauma, principalmente no tratamento de dor crônica. Estudo incluído nesta revisão observou que os participantes com dor (mecânica, inflamatória, fibromialgia e neurológica) apresentaram redução do índice de dor no grupo experimental em comparação ao grupo controle. A redução da dor foi significativamente maior no grupo de intervenção musical $(-3,4( \pm 2,3)$ e $-1,6( \pm 2,2)$, p $<0,001)$, o que correspondeu a uma melhoria relativa de $54 \%$, contra $25,8 \%$ no grupo controle. ${ }^{(36)}$ Outro estudo mostrou que 69 pacientes hospitalizados para transplante de células-tronco autólogo receberam musicoterapia, obtendo uma redução de 28\% no nível combinado de ansiedade / depressão $(\mathrm{p}=0,065)$ e $37 \%$ menor $(\mathrm{p}=0,01)$ na pontuação total dos distúrbios do humor em comparação com os controles. ${ }^{(33)}$ Estudos mostraram que o uso da música combinado com o tratamento padrão gerou maior tranquilidade com relação aos problemas crônicos de saúde nos indivíduos. ${ }^{(33,34,38,39,52)}$

Por outro lado, um ECR realizado no Canadá com 28 participantes, que teve como objetivo testar a viabilidade de uma música de baixa intensidade baseada na terapia cognitivo-comportamental para depressáo e ansiedade, mostrou que os participantes do grupo de musicoterapia, que receberam intervenção durante 9 semanas, não apresentaram diferenças estatísticas quando comparados aos participantes do grupo controle, que receberam cuidados padrão. ${ }^{(40)}$

Além disso, a presente revisão evidenciou a falta de estudos de intervenção que visam identificar os efeitos subjacentes dos mecanismos moleculares envolvidos e ativados durante a musicoterapia nos desfechos de saúde mental. Deve-se enfatizar que esta é uma área promissora e em expansão da pesquisa em enfermagem, levando o paciente ao centro do cuidado por meio da pesquisa translacional ( $\mathrm{da}$ bancada do laboratório para o lado do paciente), a fim de fornecer um atendimento personalizado aos pacientes. ${ }^{(53)}$

Mais pesquisas para avaliar o impacto da musicoterapia nos agrupamentos de sintomas na hospitalização prolongada na população adulta e para estabelecer correlaçóes com resultados clínicos devem ser incentivadas, a fim de elucidar os mecanismos subjacentes ao efeito dessa terapia complementar nos resultados de saúde. Além disso, uma avaliação mais abrangente do efeito da musicoterapia na população adulta pode ser obtida com a utilização de 
amostras maiores com ECRs bem executados, considerando também populaçóes específicas separadamente, com condiçóes agudas ou crônicas, e usando análises estatísticas multivariadas para controlar possíveis variáveis confundidoras.

Algumas limitaçóes devem ser consideradas ao interpretar os resultados desta revisão sistemática. Observou-se uma grande heterogeneidade dos estudos, utilização de diferentes estilos musicais, instrumentos musicais e tempo de seguimento. Portanto, esses achados devem ser analisados com cautela no que diz respeito à generalizaçáo dos resultados. A heterogeneidade significativa entre os estudos impediu o agrupamento dos resultados, inviabilizando a realização de meta-análise.

\section{Conclusão}

Em síntese, a musicoterapia, enquanto terapia complementar, se apresenta como potencial estratégia para reduzir a ansiedade e a depressão bem como para auxiliar na manutenção e recuperação da saúde da população adulta com diagnóstico de transtornos mentais. Ademais, os dados dessa revisão oferecem uma plataforma promissora para apoiar a investigação contínua de terapias complementares para um melhor ajuste psicológico na população adulta que sofre com transtornos mentais.

\section{Referências}

1. World Health Organization (WHO). Depression and Other Common Mental Disorders: Global Health Estimates. Geneva: WHO; 2017. [cited 2019 Jun 2]. Available from: http://www.who.int/mental_health/ management/depression/prevalence_global_healthestimates/en/

2. Penninx BW. Depression and anxiety: their insidious dance. Lancet Psychiatry. 2015;2(6):479-80.

3. Barros MB, Lima MG, Azevedo RC, Medina LB, Lopes CS, Menezes PR, et al. Depression and health behaviors in Brazilian adults - PNS 2013. Rev Saude Publica. 2017;51 Suppl 1:8s.

4. Vitorino LM, Lopes-Júnior LC, de Oliveira GH, Tenaglia M, Brunheroto A, Cortez PJ, et al. Spiritual and religious coping and depression among family caregivers of pediatric cancer patients in Latin America. Psychooncology. 2018;27(8):1900-7.

5. Zhao K, Bai ZG, Bo A, Chi I. A systematic review and meta-analysis of music therapy for the older adults with depression. Int J Geriatr Psychiatry. 2016;31(11):1188-98.
6. Zhang Y, Cai J, An L, Hui F, Ren T, Ma H, et al. Does music therapy enhance behavioral and cognitive function in elderly dementia patients? A systematic review and meta-analysis. Ageing Res Rev. 2017;35:1-11.

7. Lopes-Júnior LC, Bomfim E0, Nascimento LC, Nunes MD, Pereira-daSilva G, Lima RA. Non-pharmacological interventions to manage fatigue and psychological stress in children and adolescents with cancer: an integrative review. Eur J Cancer Care (Engl). 2016;25(6):921-35.

8. van der Steen JT, van Soest-Poortvliet MC, van der Wouden JC, Bruinsma MS, Scholten RJ, Vink AC. Music-based therapeutic interventions for people with dementia. Cochrane Database Syst Rev. 2017;5(5):CD003477.

9. Bulechek GM, Butcher HK, Dochterman JM. Classification of Nursing Interventions - NIC. 6th ed. Rio de Janeiro: Elsevier; 2016.

10. Pauwels EK, Volterrani D, Mariani G, Kostkiewics M. Mozart, music and medicine. Med Princ Pract. 2014;23(5):403-12.

11. Abrahão CA, Bomfim E, Lopes-Júnior LC, Pereira-da-Silva G. Complementary therapies as a strategy to reduce stress and stimulate immunity of women with breast cancer. J Evid Based Integr Med. 2019;24:2515690X19834169.

12. Koyama M, Wachi M, Utsuyama M, Bittman B, Hirokawa K, Kitagawa M. Recreational music-making modulates immunological responses and mood states in older adults. J Med Dent Sci. 2009;56(2):79-90.

13. Wachi M, Koyama M, Utsuyama M, Bittman BB, Kitagawa M, Hirokawa K. Recreational music-making modulates natural killer cell activity, cytokines, and mood states in corporate employees. Med Sci Monit. 2007;13(2):CR57-70.

14. Bittman B, Croft DT Jr, Brinker J, van Laar R, Vernalis MN, Ellsworth DL. Recreational Music-Making alters gene expression pathways in patients with coronary heart disease. Med Sci Monit. 2013;19:139 47.

15. Bradt J, Dileo C, Magill L, Teague A. Music interventions for improving psychological and physical outcomes in cancer patients. Cochrane Database Syst Rev. 2016;15(8):CD006911.

16. Melo GA, Rodrigues AB, Firmeza MA, Grangeiro AS, Oliveira PP, Caetano JA. Musical intervention on anxiety and vital parameters of chronic renal patients: a randomized clinical trial. Rev Lat Am Enfermagem. 2018;26(0):e2978.

17. Batt-Rawden K. The role of music in a salutogenic approach to health. Int J Ment Health Promot. 2010;12(2):11-8.

18. Brrasil. Ministério da Saúde. Portaria n. 849, de 27 de março 2017. Inclui a Arteterapia, Ayurveda, Biodança, Dança Circular, Meditação, Musicoterapia, Naturopatia, Osteopatia, Quiropraxia, Reflexoterapia, Reiki, Shantala, Terapia Comunitária Integrativa e Yoga à Política Nacional de Práticas Integrativas e Complementares. Diário Oficial da União. 2017 Mar. 28; Sec. 1:68. [citado 2021 Mai 26]. Disponível em:http://189.28.128.100/dab/docs/portaldab/documentos/ prt_849_27_3_2017.pdf

19. Aalbers S, Fusar-Poli L, Freeman RE, Spreen M, Ket JC, Vink AC, et al. Music therapy for depression. Cochrane Database Syst Rev. 2017;11(11):CD004517.

20. Moher D, Liberati A, Tetzlaff J, Altman DG; PRISMA Group. Preferred reporting items for systematic reviews and meta-analyses: the PRISMA statement. PLOS Med. 2009;(7):e1000097.

21. Methley AM, Campbell S, Chew-Graham C, McNally R, Cheraghi-Sohi S. PICO, PICOS and SPIDER: a comparison study of specificity and sensitivity in three search tools for qualitative systematic reviews. BMC Health Serv Res. 2014;14(1):579. 
22. Lopes-Júnior CL, Cruz LA, Leopoldo VC, Campos FR, Almeida AM, Silveira RC. Effectiveness of traditional chinese acupuncture versus sham acupuncture: a systematic review. Rev Lat Am Enfermagem. 2016;24(0):e2762.

23. Nunes MD, Bomfim E, Olson K, Lopes-Junior LC, Silva-Rodrigues FM, Garcia de Lima RA, et al. Interventions minimizing fatigue in children/ adolescents with cancer: an integrative review. J Child Health Care. 2018;22(2):186-204.

24. Gonçalves CA, Lopes-Júnior LC, Nampo FK, Zilly A, Mayer PC, Pereirada-Silva G. Safety, efficacy and immunogenicity of therapeutic vaccines in the treatment of patients with high-grade cervical intraepithelial neoplasia associated with human papillomavirus: a systematic review protocol. BMJ Open. 2019;9(7):e026975.

25. Lopes-Júnior LC, Bomfim E, Olson K, Neves ET, Silveira DS, Nunes $M D$, et al. Effectiveness of hospital clowns for symptom management in paediatrics: systematic review of randomised and non-randomised controlled trials. BMJ. 2020;371:m4290.

26. Galvão CM, Sawada NO, Trevizan MA. Revisão sistemática: recurso que proporciona a incorporação das evidências na prática da enfermagem. Rev Lat Am Enfermagem. 2004;12(3):549-56.

27. Silva Junior FJ, Sales JC, Monteiro CF, Costa AP, Campos LR, Miranda $\mathrm{PI}$, et al. Impact of COVID-19 pandemic on mental health of young people and adults: a systematic review protocol of observational studies. BMJ Open. 2020;10(7):e039426.

28. Lopes-Júnior LC, Rosa GS, Pessanha RM, Schuab SI, Nunes $\mathrm{KZ}$, Amorim MH. Efficacy of the complementary therapies in the management of cancer pain in palliative care: A systematic review. Rev Lat Am Enfermagem. 2020;28:e3377.

29. de Campos Pereira Silveira RC, Dos Reis PE, Ferreira EB, Braga FT, Galvão CM, Clark AM. Dressings for the central venous catheter to prevent infection in patients undergoing hematopoietic stem cell transplantation: a systematic review and meta-analysis. Support Care Cancer. 2020;28(2):425-38.

30. Lopes-Júnior LC, Rosa MA, Lima RA. Psychological and psychiatric outcomes following picu admission: a systematic review of cohort studies. Pediatr Crit Care Med. 2018;19(1):e58-67.

31. Jadad AR, Moore RA, Carroll D, Jenkinson C, Reynolds DJ, Gavaghan DJ, et al. Assessing the quality of reports of randomized clinical trials: is blinding necessary? Control Clin Trials. 1996;17(1):1-12.

32. Higgins JP, Green S. Cochrane Handbook for Systematic Reviews of Interventions. Version 5.1. Cochrane Collaboration; 2011.

33. Cassileth BR, Vickers AJ, Magill LA. Music therapy for mood disturbance during hospitalization for autologous stem cell transplantation: a randomized controlled trial. Cancer. 2003;98(12):2723-9.

34. Choi AN, Lee MS, Lim HJ. Effects of group music intervention on depression, anxiety, and relationships in psychiatric patients: a pilot study. J Altern Complement Med. 2008;14(5):567-70.

35. Erkkilä J, Punkanen M, Fachner J, Ala-Ruona E, Pöntiö I, Tervaniemi M, et al. Individual music therapy for depression: randomised controlled trial. Br J Psychiatry. 2011;199(2):132-9.

36. Guétin $\mathrm{S}$, Giniès $\mathrm{P}$, Siou DK, Picot MC, Pommié C, Guldner E, et al. The effects of music intervention in the management of chronic pain: a singleblind, randomized, controlled trial. Clin J Pain. 2012;28(4):329-37.

37. Gold C, Assmus J, Hjørnevik K, Qvale LG, Brown FK, Hansen AL, et al. Music therapy for prisoners: pilot randomised controlled trial and implications for evaluating psychosocial interventions. Int J Offender Ther Comp Criminol. 2014;58(12):1520-39.
38. Salehi B, Salehi M, Nsirnia K, Soltani P, Adalatnaghad M, Kalantari N, et al. The effects of selected relaxing music on anxiety and depression during hemodialysis: A randomized crossover controlled clinical trial study. Arts Psychother. 2016;48:76-80.

39. Dóro CA, Neto JZ, Cunha R, Dóro MP. Music therapy improves the mood of patients undergoing hematopoietic stem cells transplantation (controlled randomized study). Support Care Cancer. 2017;25(3):10138.

40. Trimmer C, Tyo R, Pikard J, McKenna C, Naeem F. Low-intensity cognitive behavioural therapy-based music group (CBT-music) for the treatment of symptoms of anxiety and depression: a feasibility study. Behav Cogn Psychother. 2018;46(2):168-81.

41. Rothwell PM. External validity of randomised controlled trials: "to whom do the results of this trial apply?". Lancet. 2005;365(9453):82-93.

42. Van Spall HG, Toren A, Kiss A, Fowler RA. Eligibility criteria of randomized controlled trials published in high-impact general medical journals: a systematic sampling review. JAMA. 2007;297(11):1233-40.

43. Gallagher LM, Lagman R, Rybicki L. Outcomes of music therapy interventions on symptom management in palliative medicine patients. Am J Hosp Palliat Care. 2018;35(2):250-7.

44. Keenan A, Keithley JK. Integrative review: effects of music on cancer pain in adults. Oncol Nurs Forum. 2015;42(6):E368-75.

45. Chen XJ, Hannibal N, Xu K, Gold C. Group music therapy for prisoners: protocol for a randomised controlled trial. Nord $\mathrm{J}$ Music Ther. 2014;23(3):224-41.

46. Nunes MD, Jacob E, Bomfim EO, Lopes-Junior LC, de Lima RA, FloriaSantos M, et al. Fatigue and health related quality of life in children and adolescents with cancer. Eur J Oncol Nurs. 2017;29:39-46.

47. Xavier WS, Pacheco ST, Silva LF, Nascimento LC, Lopes-Junior LC, Araújo $\mathrm{BB}$, et al. Intervenções não farmacológicas na melhoria da qualidade de vida de crianças/adolescentes oncológicos. Acta Paul Enferm 2020;33:e-APE-20190022.

48. Hyacinth O, Machado Silva-Rodrigues F, Darezzo Rodrigues Nunes M, Carlos Lopes L Jr, Fernandes A, Castanheira Nascimento L, et al. Pain experience, physical function, pain coping, and catastrophizing in children with sickle cell disease who had normal and abnormal sensory patterns. J Pain Symptom Manage. 2020;60(6):1079-91.

49. Lopes-Júnior LC, Pereira-da-Silva G, Silveira DS, Veronez LC, Santos $\mathrm{JC}$, Alonso JB, et al. The effect of clown intervention on self-report and biomarker measures of stress and fatigue in pediatric osteosarcoma inpatients: a pilot study. Integr Cancer Ther. 2018;17(3):928-40.

50. Tuinmann G, Preissler P, Böhmer H, Suling A, Bokemeyer C. The effects of music therapy in patients with high-dose chemotherapy and stem cell support: a randomized pilot study. Psychooncology. 2017;26(3):377-84

51. Lopes-Júnior LC, Silveira DS, Olson K, Bomfim EO, Veronez LC, Santos $\mathrm{JC}$, et al. Clown intervention on psychological stress and fatigue in pediatric patients with cancer undergoing chemotherapy. Cancer Nurs. 2020;43(4):290-9.

52. Messika J, Hajage D, Panneckoucke N, Villard S, Martin Y, Renard $E$, et al. Effect of a musical intervention on tolerance and efficacy of non-invasive ventilation in the ICU: study protocol for a randomized controlled trial. Trials. 2016;17(1):450.

53. Lopes-Júnior LC, Olson K, de Omena Bomfim E, Pereira-da-Silva G, Nascimento LC, de Lima RA. Translational research and symptom management in oncology nursing. Br J Nurs. 2016;25(10):S12, S14, S16 passim. 